\title{
Comparing human epidermal growth factor receptor 2 amplification and expression using immunohistochemistry and silver in situ hybridisation in gastric carcinoma and lymph node metastasis
}

\author{
GÜLSÜN GÜLTEN ${ }^{1}$, BAYRAM YILMAZ ${ }^{2}$ and NEŞE ÇALLI DEMIRKAN ${ }^{3}$ \\ ${ }^{1}$ Department of Pathology, Şanlıurfa Training and Research Hospital, 63250 Şanlıurfa; \\ ${ }^{2}$ Department of Pathology, Hitit University Erol Olçok Training and Research Hospital, 19040 Çorum; \\ ${ }^{3}$ Department of Pathology, Faculty of Medicine, Pamukkale University, 20160 Denizli, Turkey
}

Received October 31, 2019; Accepted May 13, 2020

DOI: $10.3892 / \mathrm{ol} .2020 .11731$

\begin{abstract}
Detecting the amplification and expression of human epidermal growth factor receptor (HER2) is important for planning trastuzumab treatment for patients with gastric carcinoma. The present study aimed to analyse HER2 amplification and expression in primary gastric adenocarcinoma tumours and metastatic lymph nodes using microarray methods, and to assess the potential contribution of these methods to treatment planning. In total, 60 patients with lymph node metastasis were included in the present study. Microarray blocks were obtained from the tissue blocks of primary tumours and metastatic lymph nodes. HER2 expression and amplification were investigated using immunohistochemical and silver in situ hybridisation (SISH) methods, respectively. Following immunohistochemical evaluation of HER2 in primary tumours, the sensitivity and specificity of the microarray method relative to the single block method were 69 and 100\%, respectively. For HER2 detection in microarray block sections from primary tumours, the sensitivity and specificity of the SISH method relative to immunohistochemistry were 56 and $100 \%$, respectively. When using SISH in microarray blocked sections, there was a high degree of concordance $(98 \%$ concordance rate) between HER2 amplification in the primary tumour and the metastatic lymph node. Furthermore, the sensitivity and specificity of metastatic lymph node results relative to those of the primary tumour were 100 and $98 \%$, respectively. Overall, the single block method was more reliable compared with the microarray method for planning treatment. When microarray
\end{abstract}

Correspondence to: Dr Gülsün Gülten, Department of Pathology, Şanlıurfa Training and Research Hospital, 1 Yenice Street, 63250 Şanlıurfa, Turkey

E-mail:dr.gulsun_88@hotmail.com

Key words: gastric carcinoma, microarray method, human epidermal growth factor receptor 2, silver in situ hybridisation blocking was used, a large number of samples must be tested to ensure reliable results. The immunohistochemical method is recommended as the first step as SISH alone increases the risk of false-negative results. Assessing HER2 amplification for treatment planning would be beneficial for primary tumours, as well as metastatic lymph nodes.

\section{Introduction}

Gastric carcinoma was reported to be the fifth most common type of malignant cancer and the third most common cause of tumour-associated mortality due to late diagnosis in the world in 2018 (1). There are contradictory reports regarding the relationship between human epidermal growth factor receptor (HER)2-positivity and the prognosis of patients with gastric carcinoma (2-4). Previously, good prognosis has been observed in patients with metastatic HER2-positive gastric carcinoma who were treated with trastuzumab, a human epidermal growth factor inhibitor $(5,6)$. HER2 gene amplification or protein overexpression has been detected in 7-30\% of primary gastric adenocarcinomas $(2,4,7-9)$. It has been reported that HER 2 amplification and overexpression are influenced by the histological type and localisation of tumours (5-10). Due to technological advances, HER2 can be easily identified; for example immunohistochemistry and fluorescence, chromogenic or silver in situ hybridisation have been used to detect HER2 protein overexpression and gene amplification $(11,12)$. Several studies have compared immunohistochemistry and in situ hybridisation as methods for detecting HER2; a high degree of agreement between the results of these methods was observed for determining HER 2 overexpression and amplification $(7-9,13)$.

Grabsch et al (14) reported HER2 immunoreactivity in $<10 \%$ of gastric carcinoma cases and $>5 \%$ of tumour cells, and demonstrated that HER2 overexpression in gastric carcinoma was heterogeneous (14). Furthermore, Hofmann et al (9) reported that heterogeneity of tumour cells was common when investigating HER2 overexpression in gastric carcinoma. In breast carcinoma, microarray methods have been useful for 
detecting HER2 amplification and overexpression (15). As heterogeneity is common in gastric carcinoma, evaluating HER 2 overexpression and amplification using the microarray method may be insufficient. In breast cancer, HER2 amplification in the primary tumour may differ from that in any metastasised lymph node tumours (16). Similarly, HER2 amplification differences in the primary tumours and metastatic lymph nodes of gastric carcinoma have also been investigated. Studies have reported a high degree of agreement between HER2 results for primary gastric tumours and synchronised lymph node metastases, although discordant cases were also observed (10,17-20).

The aims of the present study were to investigate and compare the efficacy of immunohistochemistry and silver in situ hybridisation (SISH) for detecting HER2 amplification and overexpression in the primary and metastatic lymph node tumours of gastric adenocarcinoma. In addition, HER2 expression differences were compared to assess the potential contribution of these microarray methods to treatment planning.

\section{Materials and methods}

Patients. A total of 86 patients were diagnosed with gastric adenocarcinoma using surgical resection material at the Pathology Department of Pamukkale University (Denizli, Turkey) between January 2008 and January 2014. The inclusion criteria for the present study were a diagnosis of gastric carcinoma using surgical resection material and the presence of paraffin embedded blocks for this specimen. The exclusion criteria were: i) Absence of metastases in the lymph nodes $(n=20)$; ii) spilling of tumour tissues from microarray sections of primary tumours or metastatic lymph nodes $(n=4)$; and iii) visualisation of tumour tissue in restricted areas of a limited number of metastatic lymph nodes in Node (N)1 (21) cases $(n=2)$. Hence, 60 patients were included in the present study overall. Of these, $83 \%(n=50)$ were male and $17 \%(n=10)$ were female (male/female ratio=5:1). The mean \pm standard deviation and median age of patients were $61 \pm 13$ and 63 years, respectively (range, 33-86 years). The present study was approved by The Ethics Committee for Non-Interventional Clinical Research at Pamukkale University (dated 06/12/2018; approval no. 60116787-020/47233).

Haematoxylin-eosin-stained preparations for all selected cases were prepared from formalin-fixed (using 10\% buffered formalin overnight at room temperature) paraffin-embedded tissue blocks from the archives of Pamukkale University. The blocks that best reflected the relevant tumour morphology were selected for each case. From primary tumours, 3-4 blocks, which best represented the tumour across the widest area, were selected. From metastatic lymph nodes of N2 and N3 cases, 3-4 blocks from the largest metastatic lymph nodes were selected, where tumour tissue predominated and covered most of the lymph node. In the five N1 cases included in the present study, the metastatic lymph nodes were large enough to represent the tumour in a large area.

All medical and pathologic records were reviewed to obtain patient data, including age at diagnosis, sex, tumour size, histopathological type, tumour depth, number of lymph nodes and metastatic lymph nodes. The Word Health Organisation
2010 classification (21) was used for histological classification and pathological staging.

Microarray. The tissue microarray technique (22) was used to evaluate multiple cases in a single section. The areas that best represented the tumour were selected. In N2 and N3 cases, 3-4 distinct tissue samples were obtained from either the primary tumour or metastatic lymph nodes to represent the tumour. In N1 cases, 3-4 tissue samples were obtained from distinct fields of a single block or two blocks to represent the tumour. These tissue samples, which had a 2-mm diameter, were embedded into recipient paraffin blocks. Then, 3-4- $\mu \mathrm{m}$ thick sections were collected from the recipient paraffin blocks for immunohistochemical staining and SISH.

Immunohistochemistry. Immunohistochemical staining with HER2 antibody was re-performed using the primary tumours and metastatic lymph nodes of 60 cases. The 3-4- $\mu \mathrm{m}$-thick isolated sections were dried in an oven at $60^{\circ} \mathrm{C}$ for $\geq 2 \mathrm{~h}$. The entire staining process, including deparaffinisation and antigen retrieval, was performed on a BenchMark XT fully automated immunohistochemistry-staining equipment (Ventana Medical Systems, Inc.). The tissue sections were deparaffinised using EZ Prep (Ventana Medical Systems, Inc.) at $75^{\circ} \mathrm{C}$ for $4 \mathrm{~min}$ and heat pre-treated in Cell Conditioning 1 solution (Ventana Medical Systems, Inc.) for antigen retrieval at $95^{\circ} \mathrm{C}$. Tissue sections were incubated with the anti-HER 2 rabbit monoclonal primary antibody (clone SP3; 1:100; cat. no. 237R-16; Cell Marque; Sigma-Aldrich; Merck KGaA) for $1 \mathrm{~h}$ at $37^{\circ} \mathrm{C}$ after inactivation of endogenous peroxidase activity with 3\% hydrogen peroxide (Ventana Medical Systems, Inc.) for $4 \mathrm{~min}$ at $37^{\circ} \mathrm{C}$. Non-specific antibody binding was blocked with 3\% bovine serum albumin (Ventana Medical Systems, Inc.) for $15 \mathrm{~min}$ at $37^{\circ} \mathrm{C}$. The sections were incubated with a secondary antibody followed by the application of HRP Universal Multimer for $8 \mathrm{~min}$ at $37^{\circ} \mathrm{C}$ using the ultraVIEW DAB Detection kit (Ventana Medical Systems, Inc.). The slides were counterstained with Hematoxylin II for $8 \mathrm{~min}$ at room temperature and bluing reagent for $4 \mathrm{~min}$ at room temperature. All sections were scored under a multi-head light microscope with $\mathrm{x} 40$ magnification by three pathologists (GG, YB and ÇDN) blinded to any of the clinicopathological parameters, including patient outcome. The scoring system proposed by Hofmann et al (9) was used for HER2 scoring as follows: 0, 0 or $<10 \%$ staining in tumour cells; 1 , noticeable, weak or incomplete membranous staining in $>10 \%$ of tumour cells; 2 , weak-moderate, complete or basolateral staining in $>10 \%$ of tumour cells; and 3, moderate-strong, complete or basolateral staining in $>10 \%$ of tumour cells. Scores 0 and 1 were grouped as no or low HER2 expression, while scores 2 and 3 were grouped as HER 2 positive (+). Cases with scores of 2 and 3 were considered positive for HER2 amplification. The highest HER2 score from 3-4 tissue samples, which were all prepared using the microarray block method from the primary tumours or metastatic lymph nodes of the same case, was accepted as the final score.

SISH. The SISH method was used to assess the primary tumours and metastatic lymph nodes of 60 cases. For deparaffinisation, $3-\mu \mathrm{m}$ thick sections from the microarray blocks were 
incubated in an oven at $60^{\circ} \mathrm{C}$ for $2 \mathrm{~h}$. Slices were processed in an automated BenchMark XT for SISH. After deparaffinisation with EZ Prep (Ventana Medical Systems, Inc.) at $75^{\circ} \mathrm{C}$ for $4 \mathrm{~min}$, slices were incubated in citrate buffer for $12 \mathrm{~min}$ at $90^{\circ} \mathrm{C}$ and then in ISH Protease 3 (Ventana Medical Systems, Inc.) for $16 \mathrm{~min}$ at $37^{\circ} \mathrm{C}$, denatured for $20 \mathrm{~min}$ at $80^{\circ} \mathrm{C}$ and finally hybridised for $6 \mathrm{~h}$ at $37^{\circ} \mathrm{C}$. Samples were then subjected to the SISH multimer for $16 \mathrm{~min}$, silver chromogen for $4 \mathrm{~min}$, red ISH multimer for $24 \mathrm{~min}$ and red chromogen for $8 \mathrm{~min}$, all at room temperature (all Ventana Medical Systems, Inc.). Finally, the samples were incubated with Mayer's haematoxylin for $8 \mathrm{~min}$ at room temperature and with bluing reagent for $4 \mathrm{~min}$ at room temperature to stain the background. All sections were analysed under a multi-head light microscope with $\mathrm{x} 40$ magnification by three pathologists (GG, YB and ÇDN). The HER2 gene was detected by a dinitrophenyl (DNP) labelled probe and visualized utilizing VENTANA ultraView Silver ISH DNP Detection kit (cat. no. 760-098; Ventana Medical Systems, Inc.). The chromosome-17 centromere was targeted with a digoxigenin (DIG) labelled probe and detected using VENTANA ultraView Red ISH DIG Detection kit (cat. no. 760-505; Ventana Medical Systems, Inc.). The signals for the HER2 gene and the chromosome-17 centromere were visualised in black and red, respectively; amplification was analysed by manually counting 20 consecutive cells under a light microscope with $\mathrm{x} 40$ magnification. In cases with a HER2:centromeric probe for chromosome 17 (CEP17) ratio of 1.8-2.2, 20 consecutive cells were counted again. If HER2:CEP17 ratios were $\geq 2$, these cells were considered to indicate amplification (+). An absence of amplification in 3-4 blocks prepared from the primary tumour or metastatic lymph node of the same case using the microarray block method was considered to indicate 'no amplification'. If amplification was detected in only one of the samples and not in the others, amplification was still considered to be present.

Statistical analysis. All statistical analyses were performed using SPSS version 17.0 (SPPS Inc.). The number of experimental repeats was 1 . The normal distribution of variables was examined using histograms and the Kolmogorov-Smirnov test. Mean, standard deviation, median and minimum-maximum values were used as statistical descriptors. The $\kappa$ test was used to compare HER 2 results between the primary tumours and metastatic lymph nodes, the single block method and the microarray method, and the immunohistochemical method and the SISH method. The concordance rate was calculated as the ratio of concordant cases to total cases. The predictive values of the microarray method, SISH method and metastatic lymph node HER2 results according to actual diagnoses were compared in binary groups and assessed according to sensitivity, specificity, positive predictive, negative predictive and false-negative rate values. $\mathrm{P}<0.05$ was considered to indicate a statistically significant difference.

\section{Results}

Clinicopathological features. The adenocarcinoma cases analysed in the present study were as follows: $47 \%(n=28)$ Tubular, $5 \%(n=3)$ papillary, $10 \%(n=6)$ mucinous, $35 \%(n=21)$ poorly cohesive and $3 \%(\mathrm{n}=2)$ mixed adenocarcinomas. Tumour $(\mathrm{T})$ depth was classified as follows: $2 \%(\mathrm{n}=1) \mathrm{T} 1,2 \%(\mathrm{n}=1) \mathrm{T} 2$, $18 \%(n=11)$ T3 and $78 \%(n=47)$ T4. The number of metastatic lymph nodes in cases was assessed as follows: $8 \%(n=5) \mathrm{N} 1$, $28 \%(n=17)$ N2 and 63\% $(n=38)$ were N3. Distant metastasis was present in $12 \%(n=7)$ of cases. The distribution of clinicopathological features is shown in Table I.

Comparison of immunohistochemical HER2 detection results in primary tumour sections prepared after single or microarray blocking. All 42 cases with a HER 2 score of 0 in a single block also had a score of 0 in sections prepared after microarray blocking. In five cases with a single block HER2 score of $1,40 \%(n=2)$ of these cases had a HER2 score 0 , while $60 \%(n=3)$ had score of 1 in sections prepared after microarray blocking. Moreover, five cases had a single block HER2 score of 2; however, after microarray blocking, $40 \%(n=2)$ of these cases had score $0,40 \%(n=2)$ score of 2 and $20 \%(n=1)$ score of 3. In total, eight patients had a HER2 score of 3 in a single block; after microarray blocking, 25\% $(\mathrm{n}=2)$ of these cases had a score of $0,13 \%(n=1)$ had a score of 2 and $63 \%$ $(n=5)$ had a score of 3 (Table II). For primary tumours, there was a high degree of concordance $(87 \%$ concordance rate, $\kappa=0.681, \mathrm{P}<0.001)$ between the immunohistochemical HER2 results from single blocks and sections prepared after microarray blocking. When HER 2 scores of 0 and 1 were grouped as no or low HER 2 expression and scores of 2 and 3 were grouped as HER $2^{+}$, the sensitivity of the microarray method in comparison to the single block method was $69 \%$, whereas specificity was $100 \%$, positive predictive value was $100 \%$, negative predictive value was $92 \%$ and the false-negative rate was $30 \%$ (data not shown). A case with a HER 2 score of 3 after microarray blocking is shown in Fig. 1.

Comparison of HER 2 results obtained by immunohistochemical and SISH methods using microarray blocked sections derived from primary tumours. Using the SISH method, HER2 amplification was detected in $8 \%$ of 60 cases of gastric carcinoma (data not shown). In the $9 \mathrm{HER}^{+}$cases that had HER2 scores of 2-3 according to immunohistochemistry, the SISH method detected HER 2 amplification in 56\% $(n=5)$ of cases, but not in $44 \%(n=4)$ of cases (Table III). In the four cases without HER 2 amplification, $50 \%(n=2)$ had a HER2 score of 2 and $50 \%(n=2)$ had a HER2 score of 3 according to immunohistochemistry (data not shown). Using the SISH method, HER2 amplification was not detected in $100 \%$ of the 51 no or low HER2 expression cases (HER2 score of 0-1 according to immunohistochemistry; Table III). In primary tumours, there was a high degree of concordance $(93 \%$ concordance rate, $\kappa=0.681, \mathrm{P}<0.001)$ between the HER 2 results derived from the immunohistochemical and SISH methods in microarray blocked sections. When comparing the results of the SISH method to those of the immunohistochemical method for primary tumours, sensitivity, specificity, positive predictive and negative predictive values, and the false-negative rate of the SISH method were 56, 100, 100, 93 and 44\%, respectively (data not shown).

Comparison of HER2 expression in primary tumours and metastatic lymph nodes using immunohistochemistry in microarray blocked sections. Of the 48 cases that had a HER 2 score of 0 in the primary tumour, $100 \%$ had a HER 2 score of 0 in the 
Table I. Clinicopathological features of patients with gastric carcinoma.

\begin{tabular}{lc}
\hline Clinicopathological feature & No. $(\%)$ \\
\hline Sex & \\
Male & $50(83)$ \\
Female & $10(17)$ \\
Histological type & \\
Tubular adenocarcinoma & $28(47)$ \\
Papillary adenocarcinoma & $3(5)$ \\
Mucinous adenocarcinoma & $6(10)$ \\
Poorly cohesive adenocarcinoma & $21(35)$ \\
Mixed adenocarcinoma & $2(3)$ \\
T grade & \\
1 & $1(2)$ \\
2 & $1(2)$ \\
3 & $11(18)$ \\
4 & $47(78)$ \\
N grade & \\
0 & $0(0)$ \\
1 & $5(8)$ \\
2 & $17(28)$ \\
3 & $38(63)$ \\
M grade & \\
0 & $53(88)$ \\
1 & $7(12)$ \\
\hline
\end{tabular}

T, tumour; $\mathrm{N}$, node; $\mathrm{M}$, metastasis.

metastatic lymph nodes. Of the three cases that had a HER2 score of 1 in the primary tumour, $67 \%(n=2)$ and $33 \%(n=1)$ had HER2 score of 0 and 1, respectively, in the metastatic lymph nodes. Of the three cases that had a HER 2 score of 2 in the primary tumour, $67 \%(\mathrm{n}=2)$ and $33 \%(\mathrm{n}=1)$ had HER 2 scores of 2 and 3, respectively, in the metastatic lymph nodes. Of the six cases with a HER 2 score of 3 in their primary tumour, $50 \%$ $(n=3)$ had a HER2 score of 2 and 3 in the metastatic lymph nodes (Table IV). There was a high degree of concordance ( $90 \%$ concordance rate, $\kappa=0.689, \mathrm{P}<0.001)$ between the HER 2 scores for primary tumours and those for metastatic lymph nodes when immunohistochemical testing of microarray blocked sections was used. When HER2 scores of 0 and 1 were grouped as no or low HER 2 expression, and scores of 2 and 3 were grouped as HER $2^{+}$, there was a $100 \%$ concordance rate between HER2 results for the primary tumours and those for the metastatic lymph nodes; this association was statistically significant $(\mathrm{K}=1$, $\mathrm{P}<0.001$; data not shown). Therefore, when comparing the HER2 results from the metastatic lymph nodes and the primary tumours, the sensitivity, specificity, positive predictive and negative predictive value of metastatic lymph node results were all $100 \%$ (data not shown).

Comparison of HER2 amplification in primary tumours and metastatic lymph nodes using SISH in microarray blocked sections. In $100 \%$ of the five cases with HER2 amplification

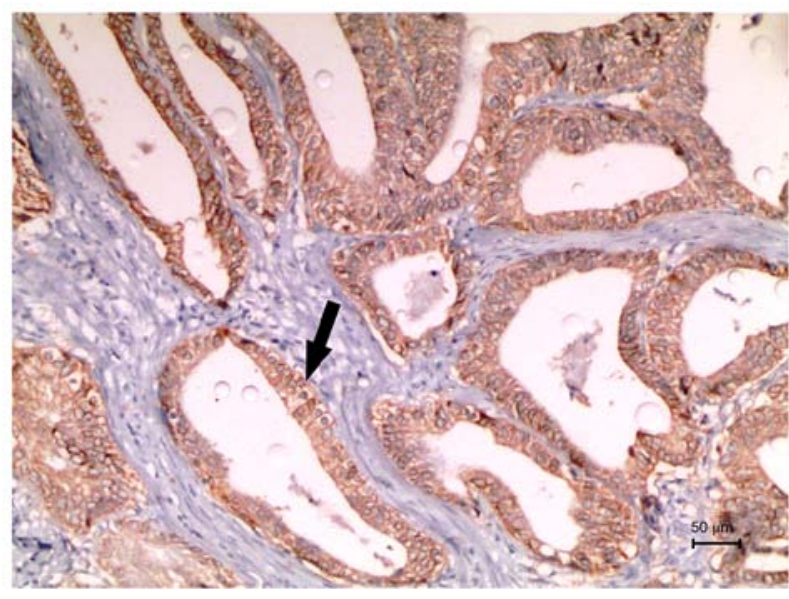

Figure 1. A gastric carcinoma case with a HER2 score of 3 after microarray blocking stained with HER 2 antibody. The arrow indicates a HER 2 positive area with clear membrane staining. Scale bar, $50 \mu \mathrm{m}$. HER2, human epidermal growth factor receptor 2.

in the primary tumour, HER2 amplification was also detected in the lymph nodes. In contrast, HER 2 amplification was detected in the lymph nodes in only $2 \%(\mathrm{n}=1)$ of the 55 cases where HER2 amplification was not detected in the primary tumours (Table V). There was a very strong concordance (98\% concordance rate, $\kappa=0.900, \mathrm{P}<0.001$ ) between the HER2 amplification in primary tumours and metastatic lymph nodes when the SISH method was used to detect HER2 amplification. When comparing the SISH-detected HER2 amplification in primary tumours and metastatic lymph nodes, the sensitivity, specificity, positive predictive and negative predictive values of the metastatic lymph node were 100, 98, 83 and $100 \%$, respectively (data not shown). A case with HER2 amplification in the primary tumour and lymph node after microarray blocking is shown in Figs. 2 and 3.

\section{Discussion}

Gastric cancer was the fifth most common type of cancer in the world in 2018 (1). Overexpression and gene amplification of $H E R 2$ has been detected in breast, stomach, colon, lung and ovarian cancer (23). Furthermore, a previous study showed that the evaluation of HER 2 was important for trastuzumab treatment in gastric carcinoma (5). However, as heterogeneity is common in gastric carcinomas, $>1$ method may be required to detect HER2 amplification and expression accurately. Differences in HER2 expression in the primary tumour and lymph node metastasis were investigated in the present study.

A wide range of prevalence has been reported for HER2 gene amplification (7-42\%) and protein expression (7-34\%) in gastric cancer (9). Such a high degree of variation may be due to the increased tumour heterogeneity in gastric carcinomas, as well as the different approaches used to assess HER2 amplification and expression. For example, Asioli et al (24), evaluated HER2 in gastric cancer with single and multiple blocks tests, and reported that HER 2 positivity increased when the latter approach was used. In another study, immunohistochemical HER2 levels in sections prepared from one and two paraffin blocks in surgically resected gastric carcinomas were 
Table II. Comparison of immunohistochemical HER2 detection results in primary tumour sections prepared after single or microarray blocking.

IHC HER2 single block score

\begin{tabular}{|c|c|c|c|c|c|c|c|c|c|c|c|}
\hline \multirow{2}{*}{$\begin{array}{l}\text { IHC HER } 2 \text { microarray } \\
\text { blocking score }\end{array}$} & \multicolumn{2}{|c|}{0} & \multicolumn{2}{|c|}{1} & \multicolumn{2}{|c|}{2} & \multicolumn{2}{|c|}{3} & \multirow[b]{2}{*}{ P-value } & \multirow[b]{2}{*}{$\kappa$} & \multirow[b]{2}{*}{$\mathrm{CR}, \%$} \\
\hline & $\mathrm{n}$ & $\%$ & $\mathrm{n}$ & $\%$ & $\mathrm{n}$ & $\%$ & $\mathrm{n}$ & $\%$ & & & \\
\hline 0 & 42 & 100 & 2 & 40 & 2 & 40 & 2 & 25 & $<0.001$ & 0.681 & 87 \\
\hline 1 & 0 & 0 & 3 & 60 & 0 & 0 & 0 & 0 & & & \\
\hline 2 & 0 & 0 & 0 & 0 & 2 & 40 & 1 & 13 & & & \\
\hline 3 & 0 & 0 & 0 & 0 & 1 & 20 & 5 & 63 & & & \\
\hline
\end{tabular}

IHC, immunohistochemistry; HER2, human epidermal growth factor receptor 2; CR, concordance rate.

Table III. Comparison of HER2 results obtained by IHC and SISH methods using microarray blocked sections derived from primary tumours.

\begin{tabular}{|c|c|c|c|c|c|c|c|}
\hline \multirow[b]{3}{*}{ Primary tumours SISH HER2 } & \multicolumn{4}{|c|}{ Primary tumours IHC HER2 } & \multirow[b]{3}{*}{ P-value } & \multirow[b]{3}{*}{$\kappa$} & \multirow[b]{3}{*}{$\mathrm{CR}, \%$} \\
\hline & \multicolumn{2}{|c|}{ Positive } & \multicolumn{2}{|c|}{$\begin{array}{c}\text { No or } \\
\text { low expression }\end{array}$} & & & \\
\hline & $\mathrm{n}$ & $\%$ & $\mathrm{n}$ & $\%$ & & & \\
\hline Amplification (+) & 5 & 56 & 0 & 0 & $<0.001$ & 0.681 & 93 \\
\hline Amplification (-) & 4 & 44 & 51 & 100 & & & \\
\hline
\end{tabular}

IHC, immunohistochemistry, SISH, silver in situ hybridisation; HER2, human epidermal growth factor receptor 2; CR, concordance rate.

Table IV. Comparison of HER2 expression in primary tumours and metastatic lymph nodes using IHC in microarray blocked sections.

\begin{tabular}{|c|c|c|c|c|c|c|c|c|c|c|c|}
\hline \multirow{3}{*}{$\begin{array}{l}\text { Metastatic lymph nodes } \\
\text { IHC HER } 2 \text { score }\end{array}$} & \multicolumn{8}{|c|}{ Primary tumours IHC HER2 score } & \multirow[b]{3}{*}{ P-value } & \multirow[b]{3}{*}{$\kappa$} & \multirow[b]{3}{*}{$\mathrm{CR}, \%$} \\
\hline & \multicolumn{2}{|c|}{0} & \multicolumn{2}{|c|}{1} & \multicolumn{2}{|c|}{2} & \multicolumn{2}{|c|}{3} & & & \\
\hline & $\mathrm{n}$ & $\%$ & $\mathrm{n}$ & $\%$ & $\mathrm{n}$ & $\%$ & $\mathrm{n}$ & $\%$ & & & \\
\hline 0 & 48 & 100 & 2 & 67 & 0 & 0 & 0 & 0 & $<0.001$ & 0.689 & 90 \\
\hline 1 & 0 & 0 & 1 & 33 & 0 & 0 & 0 & 0 & & & \\
\hline 2 & 0 & 0 & 0 & 0 & 2 & 67 & 3 & 50 & & & \\
\hline 3 & 0 & 0 & 0 & 0 & 1 & 33 & 3 & 50 & & & \\
\hline
\end{tabular}

IHC, immunohistochemistry; HER2, human epidermal growth factor receptor 2; CR, concordance rate.

compared; HER 2 positivity was $14.4 \%$ in sections prepared from one paraffin block but $19.3 \%$ in sections prepared from two paraffin blocks (25).

In gastric carcinomas, adequate and accurate histological evaluation of endoscopic biopsy materials requires 6-8 endoscopic biopsies (26). Tominaga et al (27) reported that an agreement between the resection material and biopsies in gastric carcinomas could be achieved using $\geq 5$ biopsies (27). The use of the microarray method for HER 2 evaluation in gastric carcinomas is controversial because of the high frequency of intratumoral heterogeneity in gastric cancer. For example, using gastrectomy material, Warneke et al (28) compared HER2 overexpression in a microarray prepared from five sections and in whole tissue samples prepared from 
Table V. Comparison of HER2 amplification in primary tumours and metastatic lymph nodes using SISH in microarray blocked sections.

Primary tumours SISH HER2

\begin{tabular}{|c|c|c|c|c|c|c|c|}
\hline \multirow[b]{3}{*}{ Metastatic lymph nodes SISH HER2 } & \multirow{2}{*}{\multicolumn{2}{|c|}{ Amplification (+) }} & \multirow{2}{*}{\multicolumn{2}{|c|}{ Amplification (-) }} & & & \\
\hline & & & & & \multirow[b]{2}{*}{ P-value } & \multirow[b]{2}{*}{$\kappa$} & \multirow[b]{2}{*}{$\mathrm{CR}, \%$} \\
\hline & $\mathrm{n}$ & $\%$ & $\mathrm{n}$ & $\%$ & & & \\
\hline Amplification (+) & 5 & 100 & 1 & 2 & $<0.001$ & 0.900 & 98 \\
\hline Amplification (-) & 0 & 0 & 54 & 98 & & & \\
\hline
\end{tabular}

IHC, immunohistochemistry; SISH, silver in situ hybridisation; HER2, human epidermal growth factor receptor 2; CR, concordance rate.

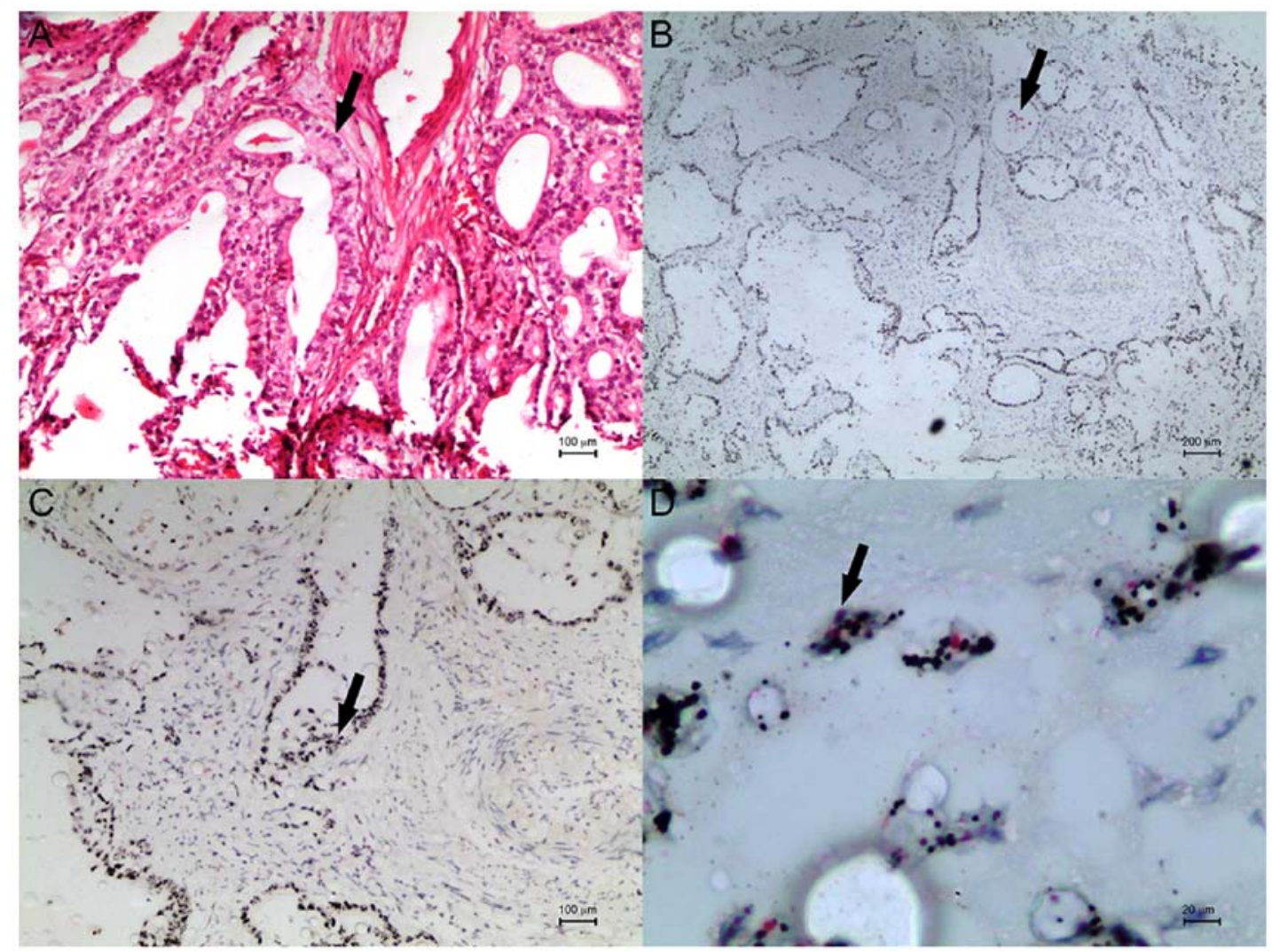

Figure 2. A gastric carcinoma case with HER2 amplification in the primary tumour after microarray blocking. (A) Haematoxylin and eosin staining. Scale bar, $100 \mu \mathrm{m}$. The arrow indicates a tumour gland in the primary tumour. (B) SISH. Scale bar, $200 \mu \mathrm{m}$. (C) SISH. Scale bar, $100 \mu \mathrm{m}$. (D) SISH. Scale bar, $20 \mu \mathrm{m}$. The arrows indicate tumour cells with a HER2:CEP17 ratio $\geq 2$. SISH, silver in situ hybridisation; HER2, human epidermal growth factor receptor 2; CEP17, centromeric probe for chromosome 17.

a single block; the group reported a false-negative rate of $24 \%$ for HER 2 overexpression when the microarray method was used. In addition, Stahl et al (29) demonstrated that HER2 amplification and overexpression results may be inaccurate when small biopsies and the microarray method are used because of the heterogeneity of gastric carcinomas. This group also suggested that these inaccuracies could potentially limit treatment decisions.

In the present study, 13 of the 60 gastric carcinoma cases had a HER 2 score 2 or 3 in the primary tumours using immunohistochemical analysis, and the HER 2 positivity rate was $22 \%$, which was consistent with the previous literature $(4,8,9)$.
To re-evaluate HER2 expression immunohistochemically for the 60 cases, the primary tumours were used to obtain new sections by blocking 3-4 tissues from each case using the microarray method, and $15 \%$ HER 2 positivity was detected in microarray blocked sections. Furthermore, in primary tumour sections, there was a high degree of concordance between HER 2 expression results from the single block method and the microarray method ( $87 \%$ concordance rate). Compared with the single block method, the sensitivity and specificity of the microarray method were 69 and $100 \%$, respectively. Additionally, the rate of false negatives for the microarray method was $30 \%$. When using the single block 


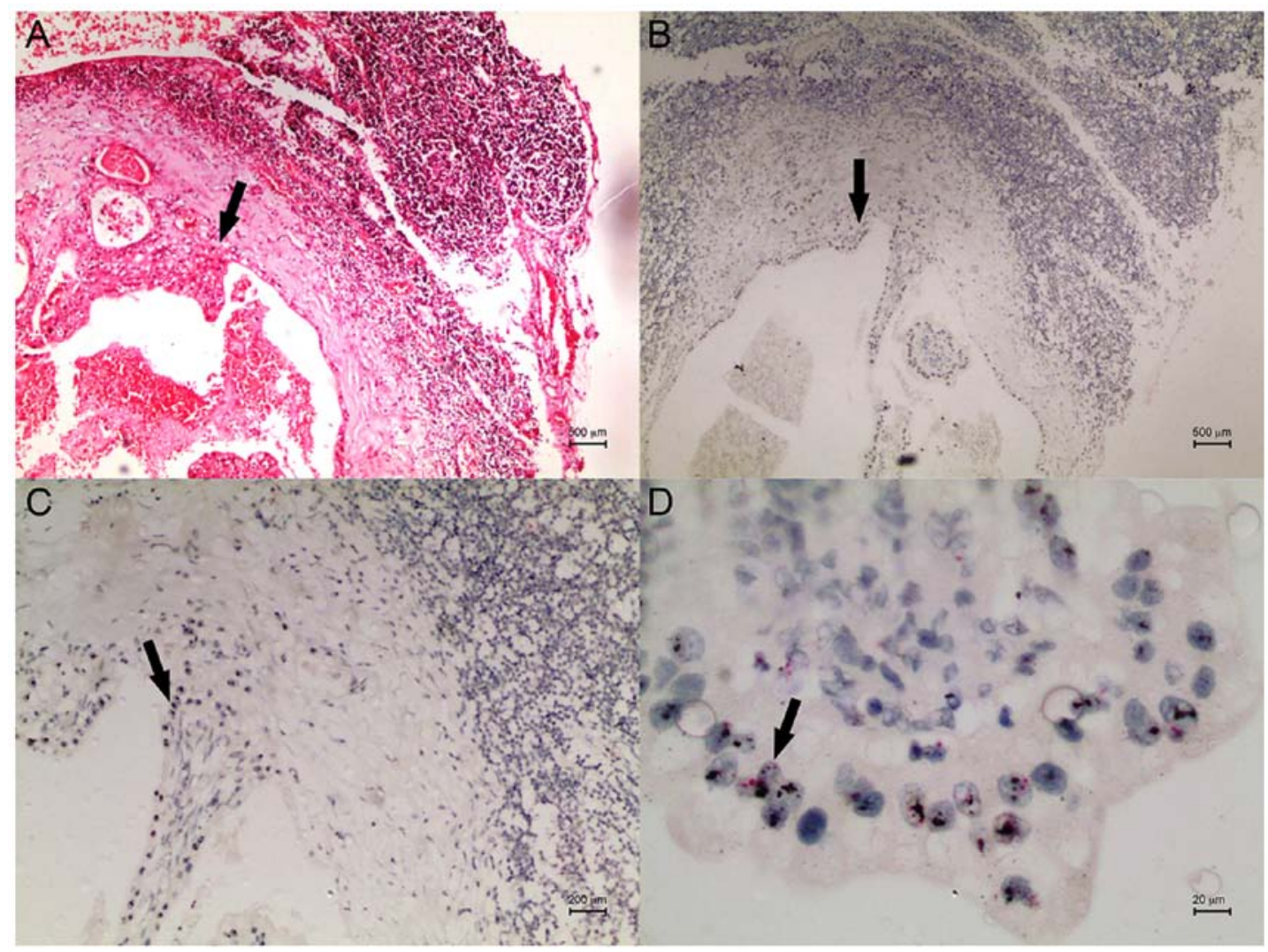

Figure 3. A gastric carcinoma case with HER2 amplification in the lymph node after microarray blocking. (A) Haematoxylin and eosin staining. Scale bar, $500 \mu \mathrm{m}$. The arrow indicates a tumour gland in the lymph node. (B) SISH. Scale bar, $500 \mu \mathrm{m}$. (C) SISH. Scale bar, $200 \mu \mathrm{m}$. (D) SISH. Scale bar, $20 \mu \mathrm{m}$. Arrows indicate tumour cells with a HER2:CEP17 ratio $\geq 2$. SISH, silver in situ hybridisation; HER2, human epidermal growth factor receptor 2; CEP17, centromeric probe for chromosome 17.

method, two cases had a HER2 score of 3 and two had a HER2 score of 2, meanwhile these cases had a HER2 score of 0 when the microarray method was used. This showed that the rate of false negatives increased because a smaller tumour area was evaluated using the microarray method. The heterogeneity of gastric carcinomas may also be a contributing factor for these discrepancies. Overall, these findings suggested that the greater the amount of tissue taken during the microarray method, the lower the rate of false negatives and the higher the number of positive cases. In addition to potentially lowering the reliability of results, the microarray method is more costly and time-consuming compared with the single block method. Therefore, with respect to time, cost and reliability, priority should be given to the single block method over the microarray method when assessing HER2 in gastric carcinoma.

Previous studies have reported a high degree of agreement between the results of immunohistochemical methods and fluorescence in situ hybridisation (FISH) for the evaluation of HER2 in gastric carcinoma $(7-9,13,17)$. Werner et al (30) investigated the agreement between HER 2 results obtained using immunohistochemical method and SISH in gastric carcinoma. The agreement was 100, 98.96, 95.83 and $96.88 \%$ in cases with an immunohistochemistry HER2 score of 0,1,2 and 3, respectively. Kim et al (31) compared the HER2 RNA-ISH method to immunohistochemistry, FISH and SISH in gastric carcinoma; the HER2 amplifications obtained using FISH or SISH methods were found to have a good agreement. In another previous study, there was an $87 \%$ agreement between HER 2 results obtained using FISH and immunohistochemistry (5). Additionally, patients who were immunohistochemically HER2- and had HER2 amplification according to the ISH method did not benefit from trastuzumab treatment, concluding that the immunohistochemical method should be the primary method for evaluation of HER2 (5).

In the present study, the SISH method was applied to microarray blocked sections from primary tumours and HER2 amplification was detected in $8 \%$ of 60 gastric carcinoma cases, which is consistent with previous literature $(2,7,9)$. In these microarray blocked sections from primary tumours, a high degree of concordance $(93 \%)$ was observed between immunohistochemical HER2 expression and HER2 amplification using the SISH method, which is consistent with previous literature $(30,31)$. When compared with the immunohistochemical method, the sensitivity and specificity of the SISH method were 56 and $100 \%$, respectively, while the false-negative rate was $44 \%$. This suggested that HER $2^{+}$cases were detected less frequently by the SISH method and no or low HER 2 expression cases were detected $100 \%$ of the time using SISH. Therefore, although there is a high degree of compatibility between the immunohistochemical and SISH methods, it was concluded that immunohistochemistry should be the first step during evaluation of HER2 positivity in gastric carcinoma treatment plans as SISH alone can produce false-negative results. 
Several studies have compared HER 2 positivity in the primary tumours and metastatic lymph nodes of gastric carcinoma. In studies by Ieni et al (19) and Fusco et al (32) the agreement between HER 2 results for primary gastric tumours and synchronised lymph node metastases were revealed to be high (95 and 90.74\%, respectively). Furthermore, Kochi et al (18) Bozzetti et al (17) and Marx et al (7) found high degrees of agreement between HER2 results obtained using immunohistochemistry and FISH in primary tumours and metastatic lymph nodes. Kochi et al (18) also suggested that analysing the primary tumour alone for HER2 levels is not always sufficient to inform treatment selection, concluding that metastatic lymph nodes should also be evaluated for HER2. Kim et al (10) reported a divergence rate of $21.8 \%$ between HER2 results obtained using immunohistochemistry in primary and metastatic tumours. Furthermore, Pagni et al (20) revealed a significant divergence between the HER 2 results from primary tumours and lymph node metastases and suggested that this diversity was due to intratumoral heterogeneity.

In the present study, when using immunohistochemistry to assess microarray blocked sections, a high degree of concordance $(90 \%)$ was observed between the HER 2 scores for primary tumours and metastatic lymph nodes. HER2 scores for primary and metastatic lymph nodes were not associated in $10 \%$ of the 60 cases; of the six discordant cases, $50 \%(n=3)$ had HER 2 score of 3 for primary tumour and HER2 score of 2 for metastatic lymph nodes. This suggested that discrepancies in HER2 score may exist between primary tumours and lymph node metastases in gastric carcinoma due to intratumoral heterogeneity. However, when the HER2 scores were grouped as either positive or no or low expression, a 100\% concordance between HER 2 results from primary tumours and those from metastatic lymph nodes was observed. Primary tumours and metastatic lymph nodes were HER $2^{+}$in nine patients, and primary tumours and metastatic lymph nodes were HER2 no or low expression in 51 patients. Therefore, although differences in HER2 scores existed, complete agreement for HER2 positivity in primary tumours and metastatic lymph nodes was observed when HER2 scores of 0 and 1 were grouped as no or low HER 2 expression, and scores of 2 and 3 were grouped as HER $2^{+}$. Moreover, the sensitivity and specificity for detecting the metastatic lymph nodes were both $100 \%$ when compared with those of the primary tumours. Thus, immunohistochemical HER2 evaluation of primary tumours may be sufficient for planning trastuzumab treatment; however, evaluation of HER2 in metastatic lymph nodes could be used when primary tumour tissues cannot be obtained.

When using SISH in microarray blocked sections, a $98 \%$ concordance rate was observed between the HER2 amplification results from the primary tumour and those from the metastatic lymph node. In the five cases with HER2 amplification in the primary tumour, there was also HER2 amplification in the metastatic lymph nodes. Among 55 cases in which HER2 amplification was not observed in the primary tumour, only one case also had amplification in the metastatic lymph node. In this specific case, the immunohistochemical method yielded a HER 2 score of 3 in the primary tumour sections prepared from a single block. In contrast, the immunohistochemical method resulted in a HER2 score of 0 in microarray blocked sections of the primary tumour and HER2 amplification was not detected by the SISH method. These discrepancies may have been due to heterogeneity. Furthermore, in the metastatic lymph nodes of this case, the immunohistochemical method resulted in a HER2 score of 0 in the sections blocked by the microarray method. The results of this anomalous case suggested that HER2 positivity and amplification cannot always be detected in the primary tumour, but HER2 positivity and/or amplification may still be detected in the metastatic lymph nodes due to heterogeneity. Moreover, there may be cases of gastric carcinoma in which HER 2 positivity cannot be detected in metastatic lymph nodes using the immunohistochemical method but HER2 amplification can found using SISH. Therefore, it was concluded that both the immunohistochemical method and SISH method may be used for analysing HER2 amplification to identify patients suitable for trastuzumab treatment, and that this should be a focus of future work.

In conclusion, priority should be given to the single block method when evaluating HER2 by immunohistochemical staining in gastric carcinomas. The limitation of the present study was obtaining 3-4 tissue samples from the primary tumour and metastatic lymph nodes in microarray block sections. This is because microarray blocked sections are evaluated in smaller areas, and therefore, false-negative results for HER2 amplification and overexpression may be found due to intratumoral heterogeneity. To ensure trastuzumab treatment planning is reliable and effective, when using the microarray method, a large amount of tissue should be blocked and evaluated in each case. The immunohistochemical method should also be used as a first step in treatment planning as it is more accurate compared with the SISH method, which when used alone may lead to false-negative results. Finally, investigating numerous tumour areas and tumour types (both primary and lymph node metastases) using immunohistochemical and ISH methods may increase the chances of successful trastuzumab treatment, and this should be a focus of future work.

\section{Acknowledgements}

Not applicable.

\section{Funding}

No funding was received.

\section{Availability of data and materials}

All data generated or analysed during this study are included in this published article.

\section{Authors' contributions}

GG, BY and NYD contributed to the design and conception of the study, data collection, data analysis and literature research. GG, BY and NYD performed histopathological examination of gastric carcinomas. GG wrote the manuscript. All authors read and approved the final manuscript.

\section{Ethics approval and consent to participate}

The present study was approved by The Ethics Committee for Non-Interventional Clinical Research at Pamukkale 
University (dated 12.06.2018; approval no. 60116787-020/4 7233). Patient consent for participation was waived by The Ethics Committee for Non-Interventional Clinical Research at Pamukkale University.

\section{Patient consent for publication}

Not applicable.

\section{Competing interests}

The authors declare that they have no competing interests.

\section{References}

1. Bray F, Ferlay J, Soerjomataram I, Siegel RL, Torre LA and Jemal A: Global cancer statistics 2018: GLOBOCAN estimates of incidence and mortality worldwide for 36 cancers in 185 countries. CA Cancer J Clın 68: 394-424, 2018.

2. Tanner M, Hollmén M, Junttila TT, Kapanen AI, Tommola S, Soini Y, Helin H, Salo J, Joensuu H, Sihvo E, et al: Amplification of HER-2 in gastric carcinoma: Association with Topoisomerase IIalpha gene amplification, intestinal type, poor prognosis and sensitivity to trastuzumab. Ann Oncol 16: 273-278, 2005.

3. Jørgensen JT and Hersom M: HER2 as a prognostic marker in gastric cancer-a systematic analysis of data from the literature. J Cancer 3: 137-144, 2012.

4. Park DI, Yun JW, Park JH, Oh SJ, Kim HJ, Cho YK, Sohn CI, Jeon WK, Kim BI, Yoo CH, et al: HER-2/neu amplification is an independent prognostic factor in gastric cancer. Dig Dis Sci 51 1371-1379, 2006.

5. Bang YJ, Van Cutsem E, Feyereislova A, Chung HC, Shen L, Sawaki A, Lordick F, Ohtsu A, Omura Y, Satoh T, et al: ToGA Trial Investigators. Trastuzumab in combination with chemotherapy versus chemotherapy alone for treatment of HER2-positive advanced gastric or gastro-oesophageal junction cancer (ToGA): A phase 3, open-label, randomised controlled trial. Lancet 376: 687-697, 2010.

6. Cortés-Funes H, Rivera F, Alés I, Márquez A, Velasco A, Colomer R, García-Carbonero R, Sastre J, Guerra J and Grávalos C: Phase II of trastuzumab and cisplatin in patients with advanced gastric cancer with HER2/neu overexpression/amplification. J Clin Oncol 25 (Suppl 18): 4613, 2007.

7. Marx AH, Tharun L, Muth J, Dancau AM, Simon R, Yekebas E, Kaifi JT, Mirlacher M, Brümmendorf TH, Bokemeyer C, et al: HER-2 amplification is highly homogenous in gastric cancer. Hum Pathol 40: 769-777, 2009.

8. Takehana T, Kunitomo K, Kono K, Kitahara F, Iizuka H, Matsumoto Y, Fujino MA and Ooi A: Status of c-erbB-2 in gastric adenocarcinoma: A comparative study of immunohistochemistry, fluorescence in situ hybridization and enzyme-linked immuno-sorbent assay. Int J Cancer 98: 833-837, 2002.

9. Hofmann M, Stoss O, Shi D, Büttner R, van de Vijver M, Kim W, Ochiai A, Rüschoff J and Henkel T: Assessment of a HER2 scoring system for gastric cancer: Results from a validation study. Histopathology 52: 797-805, 2008.

10. Kim MA, Lee HJ, Yang HK, Bang YJ and Kim WH: Heterogeneous amplification of ERBB2 in primary lesions is responsible for the discordant ERBB2 status of primary and metastatic lesions in gastric carcinoma. Histopathology 59: 822-831, 2011

11. Francis GD, Jones MA, Beadle GF and Stein SR: Bright-field in situ hybridization for HER2 gene amplification in breast cancer using tissue microarrays: Correlation between chromogenic (CISH) and automated silver-enhanced (SISH) methods with patient outcome. Diagn Mol Pathol 18: 88-95, 2009.

12. Ross JS, Slodkowska EA, Symmans WF, Pusztai L, Ravdin PM and Hortobagyi GN: The HER-2 receptor and breast cancer: Ten years of targeted anti-HER-2 therapy and personalized medicine. Oncologist 14: 320-368, 2009.

13. Yano T, Doi T, Ohtsu A, Boku N, Hashizume K, Nakanishi M and Ochiai A: Comparison of HER2 gene amplification assessed by fluorescence in situ hybridization and HER2 protein expression assessed by immunohistochemistry in gastric cancer. Oncol Rep 15: 65-71, 2006.
14. Grabsch H, Sivakumar S, Gray S, Gabbert HE and Müller W: HER2 expression in gastric cancer: Rare, heterogeneous and of no prognostic value- conclusions from 924 cases of two independent series. Cell Oncol 32: 57-65, 2010.

15. Drev P, Grazio SF and Bracko M: Tissue microarrays for routine diagnostic assessment of HER2 status in breast carcinoma. Appl Immunohistochem Mol Morphol 16: 179-184, 2008.

16. Simon R, Nocito A, Hübscher T, Bucher C, Torhorst J, Schraml P, Bubendorf L, Mihatsch MM, Moch H, Wilber K, et al: Patterns of Her-2/neu amplification and overexpression in primary and metastatic breast cancer. J Natl Cancer Inst 93: 1141-1146, 2001.

17. Bozzetti C, Negri FV, Lagrasta CA, Crafa P, Bassano C, Tamagnini I, Gardini G, Nizzoli R, Leonardi F, Gasparro D, et al: Comparison of HER2 status in primary and paired metastatic sites of gastric carcinoma. Br J Cancer 104: 1372-1376, 2011.

18. Kochi M, Fujii M, Masuda S, Kanamori N, Mihara Y, Funada T, Tamegai H, Watanabe M, Suda $\mathrm{H}$ and Takayama T: Differing deregulation of HER2 in primary gastric cancer and synchronous related metastatic lymph nodes. Diagn Pathol 8: 191, 2013.

19. Ieni A, Barresi V, Caltabiano R, Caleo A, Bonetti LR, Lanzafame S, Zeppa P, Caruso RA and Tuccari G: Discordance rate of HER2 status in primary gastric carcinomas and synchronous lymph node metastases: A multicenter retrospective analysis. Int J Mol Sci 15: 22331-22341, 2014.

20. Pagni F, Zannella S, Ronchi S, Garanzini C and Leone BE: HER2 status of gastric carcinoma and corresponding lymph node metastasis. Pathol Oncol Res 19: 103-109, 2013.

21. Bosman FT, Carneiro F, Hruban RH and Theise ND: World Health Organisation classification of tumors of the digestive system. IARC, Lyon, 2010.

22. Simon R, Mirlacher $M$ and Sauter G: Tissue microarrays Methods Mol Med 114: 257-268, 2005.

23. David L, Seruca R, Nesland JM, Soares P, Sansonetty F, Holm R, Børresen AL and Sobrinho-Simões M: C-erbB-2 expression in primary gastric carcinomas and their metastases. Mod Pathol 5: 384-390, 1992.

24. Asioli S, Maletta F, Verdun di Cantogno L, Satolli MA, Schena M, Pecchioni C, Botta C, Chiusa L, Molinaro L, Conti L, et al: Approaching heterogeneity of human epidermal growth factor receptor 2 in surgical specimens of gastric cancer. Hum Pathol 43: 2070-2079, 2012.

25. Ge X, Wang H, Zeng H, Jin X, Sujie A, Xu C, Liu Y, Huang J, Ji Y, Tan Y, et al: Clinical significance of assessing Her2/neu expression in gastric cancer with dual tumor tissue paraffin blocks. Hum Pathol 46: 850-857, 2015.

26. National Comprehensive Cancer Network clinical practice guidelines in oncology (version 2.2015): Gastric cancer.

27. Tominaga N, Gotoda T, Hara M, Hale MD, Tsuchiya T, Matsubayashi J, Kono S, Kusano C, Itoi T, Fujimoto K, et al: Five biopsy specimens from the proximal part of the tumor reliably determine HER2 protein expression status in gastric cancer. Gastric Cancer 19: 553-560, 2016.

28. Warneke VS, Behrens HM, Böger C, Becker T, Lordick F, Ebert MPA and Röcken C: Her2/neu testing in gastric cancer: Evaluating the risk of sampling errors. Ann Oncol 24: 725-733, 2013.

29. Stahl P, Seeschaaf C, Lebok P, Kutup A, Bockhorn M, Izbicki JR, Bokemeyer C, Simon R, Sauter G and Marx AH: Heterogeneity of amplification of HER2, EGFR, CCND1 and MYC in gastric cancer. BMC Gastroenterol 15: 7, 2015.

30. Werner D, Battmann A, Steinmetz K, Jones T, Lamb T, Martinez M, Altmannsberger HM and Al-Batran SE: The validation of a novel method combining both HER2 immunohistochemistry and HER2 dual-colour silver in situ hybridization on one slide for gastric carcinoma testting. J Transl Med 12: 160, 2014.

31. Kim MA, Jung JE, Lee HE, Yang HK and Kim WH: In situ analysis of HER 2 mRNA in gastric carcinoma: Comparison with fluorescence in situ hybridization, dual-color silver in situ hybridization, and immunohistochemistry. Hum Pathol 44: 487-494, 2013

32. Fusco N, Rocco EG, Del Conte C, Pellegrini C, Bulfamante G, Nuovo FD, Romagnoli $S$ and Bosari S: HER2 in gastric cancer: A digital image analysis in pre-neoplastic, primary and metastatic lesions. Mod Pathol 26: 816-824, 2013.

This work is licensed under a Creative Commons Attribution-NonCommercial-NoDerivatives 4.0 International (CC BY-NC-ND 4.0) License. 